\title{
HIGH RESOLUTION NIR IMAGING OF THE STARBURST GALAXY NGC 1808
}

\author{
L.E. TACCONI-GARMAN and A. KRABBE \\ Max-Planck-Institut für extraterrestrische Physik \\ A. STERNBERG \\ Tel Aviv University \\ and \\ R. GENZEL \\ Max-Planck-Institut für extraterrestrische Physik
}

\begin{abstract}
We report 0.6" res. J, $\mathrm{H}$, and $\mathrm{K}$ and 1.5" res. imaging of $2.17 \mu \mathrm{m} \mathrm{HI} \mathrm{Br} \gamma$ and $2.12 \mu \mathrm{m}$ $\mathrm{H}_{2}$ 1-0 S(1) line emission towards the nucleus of the starburst galaxy NGC 1808. In the Kband data we (partially) resolve the nucleus and see several small knots in the circumnuclear region. Further, our JHK continuum images show that a large fraction of the near infrared light in NGC 1808 is produced in young star forming clusters. The $\mathrm{Br} \gamma$ emission originates from a compact nuclear source and from several distinct emission knots in the circumnuclear region. These knots are spatially well correlated with a family of compact radio sources, but uncorrelated with the optical "hot spots". We propose that the $\mathrm{Br} \gamma$ knots trace the actual sites of starburst activity, while the optical hot spots are just directions of low foreground extinction.

We use our data together with radio and far-infrared continuum emission measurements to constrain the parameters of the individual starburst sites in NGC 1808. The data suggest that the starbursts are unsynchronized and prolonged $\left(5 \times 10^{6}-5 \times 10^{7} \mathrm{yrs}\right)$. The star formation rates in the active sites range from $\sim 0.1$ to $\sim 0.6 \mathrm{M}_{\odot} \mathrm{yr}^{-1}$, and the present rapid rate of star-formation in NGC 1808 can be maintained for at most another $\sim 7 \times 10^{7} \mathrm{yrs}$.

Portions of this work are presently in press (Krabbe, Sternberg, and Genzel 1993), and a second paper is in preparation (Tacconi-Garman et al. 1993).
\end{abstract}

\section{References}

Krabbe, A., Sternberg, A., and Genzel, R.: 1993, 'Near Infrared Spectral Imaging of NGC 1808:

Probing the Starburst', Astrophys. J., in press

Tacconi-Garman, L.E., et al.: 1993, in preparation.

461

T. J.-L. Courvoisier and A. Blecha: Multi-Wavelength Continuum Emission of AGN, 461.

(C) 1994 IAU. Printed in the Netherlands. 